Document downloaded from:

http://hdl.handle.net/10251/43345

This paper must be cited as:

Mendes, LDP.; Rodrigues, JJPC.; Lloret, J.; Sendra Compte, S. (2014). Cross-layer Dynamic Admission Control for Cloud-based Multimedia Sensor Networks. IEEE Systems Journal. 8(1):235-246. doi:10.1109/JSYST.2013.2260653.

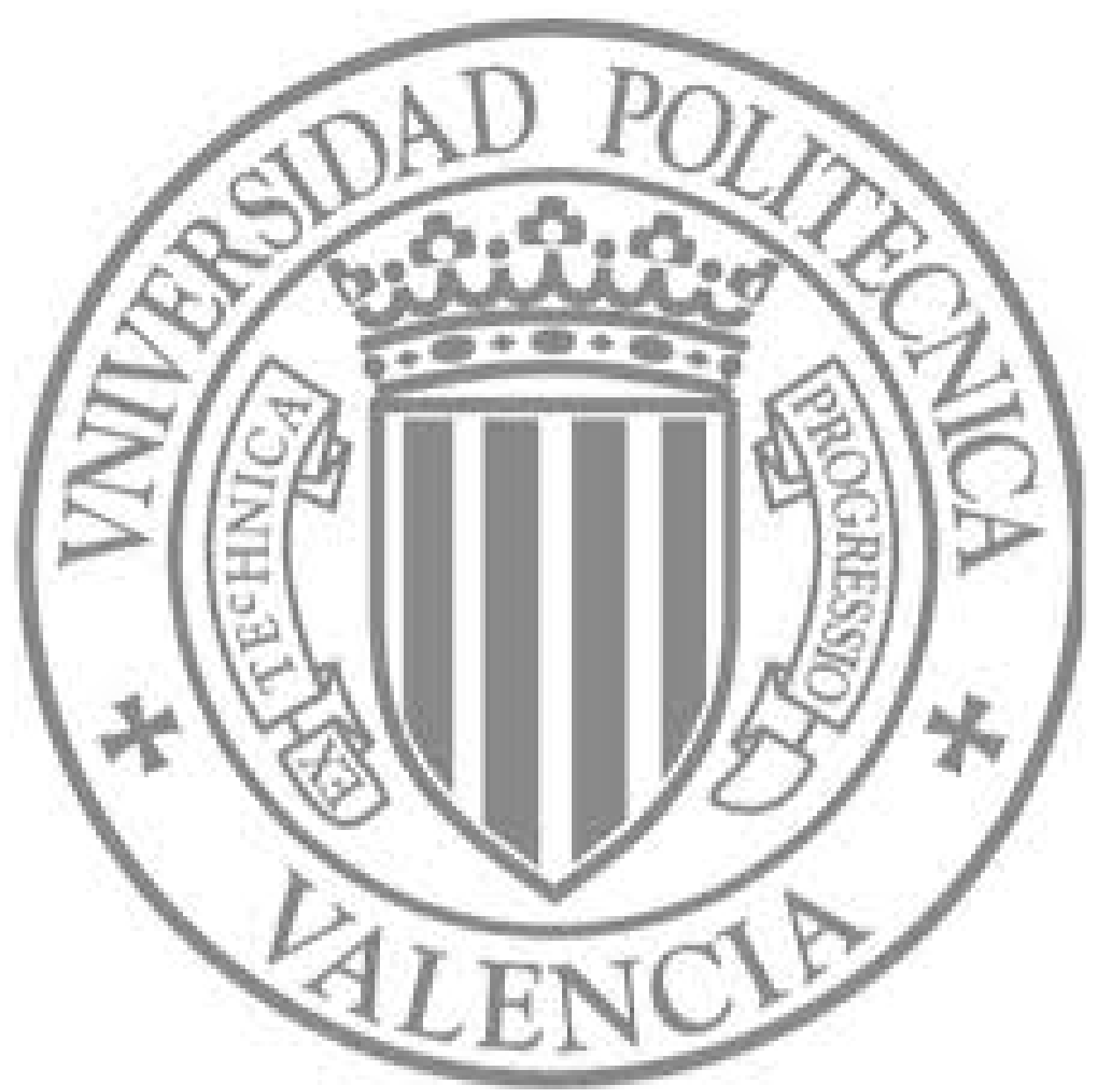

The final publication is available at

http://dx.doi.org/10.1109/JSYST.2013.2260653

Copyright Institute of Electrical and Electronics Engineers (IEEE) 


\title{
Cross-layer Dynamic Admission Control for Cloud-based Multimedia Sensor Networks
}

\author{
Lucas D. P. Mendes, Joel J. P. C. Rodrigues, Jaime Lloret, Sandra Sendra
}

\begin{abstract}
Cloud-based communications system is now widely used in many application fields such as medicine, security, and environment protection, etc. Its use is being extended to the most demanding services like multimedia delivery. However, there are a lot of constraints when cloud-based sensor networks use the standard IEEE 802.15.3 or IEEE 802.15.4 technologies. This paper proposes a channel characterization scheme combined to a cross-layer admission control in a dynamic cloud-based multimedia sensor networks to share the network resources among any two nodes. The analysis shows the behaviour of two nodes using different network access technologies and the channel effects for each technology. Moreover, it is also shown the existence of optimal node arrival rates in order to improve the usage of dynamic admission control when network resources are used. An extensive simulation study was performed to evaluate and validate the efficiency of the proposed dynamic admission control for cloudbased multimedia sensor networks.
\end{abstract}

\section{Index Terms}

Cross-layer Design, admission control, multimedia communications, cloud computing, sensor networks.

L. D. P. Mendes and J. J. P. C. Rodrigues are with the Instituto de Telecomunicações, University of Beira Interior, Portugal (email: lucas.mendes@it.ubi.pt, joeljr@ieee.org); J. Lloret is with the Universidad Politecnica de Valencia, Spain (email: jlloretedcom.upv.es); Sandra Sendra is with the Universidad Politecnica de Valencia, Spain (email: sansenco@posgrado.upv.es). 


\section{INTRODUCTION}

Ever since small devices with sensing capabilities and wireless transmitters have been developed, the number of applications for these devices has substantially increased. Wireless Sensor Networks (WSNs) deployments started in the military field [?], but they have also reached the medical field (e.g., in patient monitoring and health problems detection applications), the industry (e.g., to monitor production processes and make them more efficient), and also enabled remote monitoring (e.g., for environmental protection purposes or security).

Although new spheres of applications could be deployed thanks to these small devices, new challenges needed to be addressed in order to turn the use of WSNs viable. The main limitation of sensor nodes is their reduced energy capacity, making necessary the deployment of specific protocols to keep the control messages transmission (overhead) at a minimum [?]. Some solutions have arised to overcome these limitations, such as channel-adaptive transmission [?] and energy harvesting [?]. However, it has already been identified that solutions considering a single layer from the protocol stack can only yield limited gains [?]. Thus, cross-layer design [?] has emerged and further improvements could be added to WSNs, as shown in the following examples. Data aggregation can reduce the amount of redundant transmission in the network. Thus, Al-Karaki et al. [?] proposed GRASS protocol which divides the network in clusters and calculates the most energy-efficient routes with data aggregation to the sink, providing at least $35 \%$ lifetime improvement over the directed diffusion protocol. Furthermore, new medium access methods have been also proposed. Ren and Liang proposed the throughput maximized medium access control (TM-MAC) [?] which can determine the optimal transmission schedule and rate in order to increase throughput in IEEE 802.15.3 networks, and Kim and Park proposed the transport controlled MAC (TC-MAC) [?] which uses channel reservation to reduce end-to-end delay and explicit congestion notifications to reduce network congestion. Hence, from the last two examples, it can be seen that medium access methods are still being created and modified since there is no method proven to be the most efficient. Most of them are based on ALOHA, time division multiple access (TDMA), or carrier sense multiple access (CSMA). Although the simplicity of ALOHA can provide network throughput gains [?], mainly when it is compared with CSMA, we can see that has more overhead due to its medium contention scheme. However, TDMA can guarantee quality of service $(\mathrm{QoS})$, needed for multimedia transmission. 
The specific challenge of communicating different node technologies in the same WSN is tackled in this work. In order to study this challenge, a military scenario is proposed. Any other scenario could be considered for this evaluation, without changing the validity of the proposal. Hence, based on a comprehensive channel characterization presented in Section 4, emphasizing the channel differences that affect IEEE 802.15.3 [?] and IEEE 802.15.4 [?] sensor nodes, a dynamic TDMA method with a cross-layer admission control is proposed to share the network resources between the two considered types of nodes in the cloud-based multimedia WSN. The achieved results show the channel effects for each technology, as well as the influence of the nodes interarrival time on the use of network resources. The IEEE 802.15.5 presents an evolution of IEEE 802.15.4 that includes mesh routing [?] [?]. We have not considered it in this work because packet forwarding is not necessary in the considered scenarios.

In the related literature there are very few works on cloud-based multimedia sensor networks. In [?], M. M. Hassan et al., bearing in mind to facilitate connecting sensors, people and software objects to build community-centric sensing applications, they proposed a framework to integrate sensor networks to the emerging data center cloud model of computing. Moreover, W. Zhu et al. tackle the idea of multimedia cloud computing in [?] and present a framework where wireless sensor networks are taken into account. But the most related works with this paper are [?], by $\mathrm{K}$. Lee et al., that propose the use of a cloud computing model for an integrated environmental monitoring an modeling applications, and [?], where W. Kurschl et al. present a model, which combines the concept of wireless sensor networks with the cloud computing paradigm, and show how both can benefit from this combination.

The remainder of this paper is organized as follows. In Section 2, the system model and some of the scenario considerations are explained. Wireless channel characterization is made in Section 3. In Section 4, the dynamic TDMA method is proposed and the cross-layer admission control is explained. Section 5 presents and discusses the results achieved by the proposal in the considered scenario. Section 6 shows the comparison of this proposal with other admission control systems used in IEEE 802.15.3 and IEEE 802.15.4. Finally, Section 7 concludes the paper and suggests directions for future work. 


\section{SySTEM MODEL}

WSN is considered to operate in a military surveillance scenario. In order to prevent enemy troops from invading a determined area, surveillance is necessary. Ideally, hidden cameras should be deployed to provide full surveillance of the protected area without risking soldiers lives. However, nodes with multimedia capabilities are expensive and cannot be wasted. Thus, other simpler and cheaper support sensors should be deployed alongside the multimedia networks. In this work, it is considered that two types of sensor nodes can be deployed to analyze enemy troops movements - multimedia nodes, with characteristics from the IEEE 802.15.3 technology, and movement and light intensity sensor nodes, with characteristics from the IEEE 802.15.4 technology. This example scenario will allow the analysis of the network behavior when different types of nodes are present, which can be extended to other technologies and applications. Other applications for the considered heterogeneous network, adjusting the nodes deployment as necessary, are intrusion detection systems, e.g., the border surveillance proposal presented by Kosar et al. in [?].

Although sensors are usually deployed from air planes in applications where sensors are left unassisted, such as military and disaster scenarios [?], in this work, troops will deploy new nodes and possibly recover nodes without battery that have not been destroyed by the enemy. These sensor nodes are deployed randomly, according to an exponential distribution and the $x$ and $y$-coordinate of deployment are each selected from a uniform distribution ranging from 0 to the network size. Due to the considered channel characteristics explained in next section, some nodes might not be able to connect to the sink node after the deployment, and thus they are considered to be collected and redeployed later. Figure 1 shows a deployment example with both kinds of nodes, with a square-shaped network with edge length of $500 \mathrm{~m}$.

The sensors energy constraints are modelled as the maximum number of frames they can transmit before there is no energy left for transmission. Since multimedia sensors have a greater transmission rate than the scalar sensors (11Mbps against 250kbps) and since the former will transmit larger frames than the latter, multimedia sensors will transmit less frames than the scalar ones before exhausting their batteries.

Although there will be different distances between the sensors and sink, which would lead to different propagation delays, these delays will be considered to be zero in order to simplify 


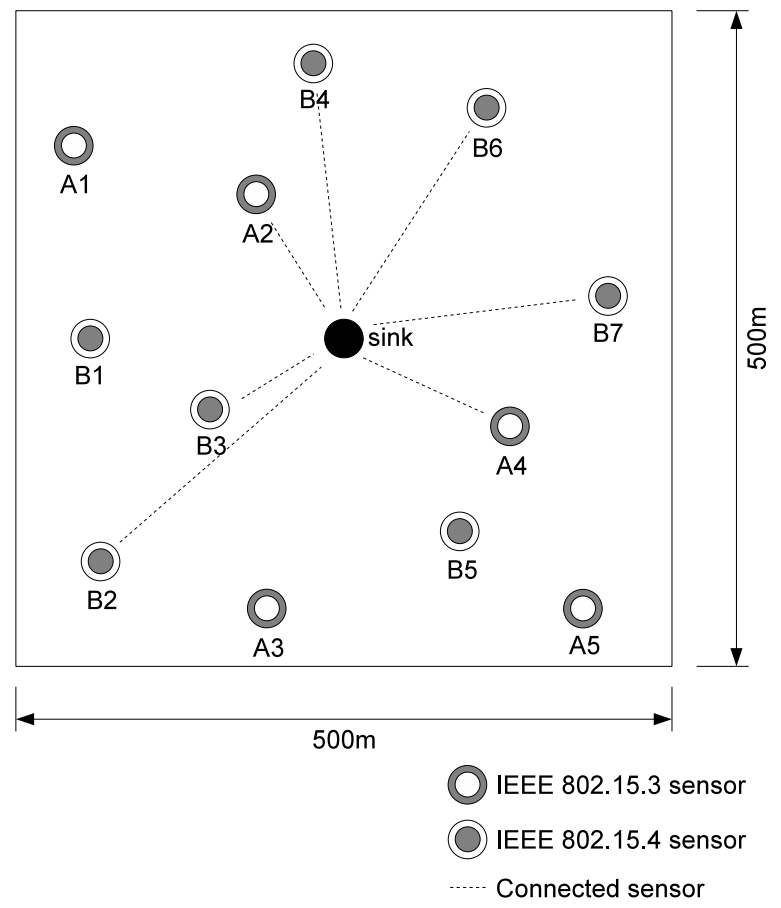

Fig. 1. Example of nodes deployment.

synchronism. In a real deployment, the use of guard bits for each transmitted frame, enough to counteract the effects of the worst case propagation delay, might solve this part of the synchronization problem.

Multimedia transmitted by the corresponding type of sensors is limited by frame maximum delay. Hence, this application constraint will be used to limit new nodes admission at the link layer, causing some recently deployed nodes to be blocked. Thus, the stricter the constraints, the less nodes will be allowed to connect to the sink and transmit.

\section{Channel Considerations}

Regarding the presented system model, a stochastic channel model can be used to determine if incoming nodes will be able to connect to the sink node. Since the sensors will be deployed on building walls or rooftops, the two main signal attenuation effects will be the path loss and shadowing (caused by obstacles such as other buildings between a sensor and its sink node). Many outdoor empirical models have been defined, and some of them are the LongleyRice model, Durkin's model, Okumura model, Hata model, personal communications systems 
(PCS) extension to Hata model (or COST 231 model), Walfisch and Bertoni model, wideband PCS microcell model, and the linear piecewise multi-slope model [?] [?]. Nevertheless, all these models have been formulated focusing cellular phone systems, and thus they rely on information on height of transmission towers and consider transmission spanning kilometers of distance. Hence, a simplified stochastic path loss and shadowing model (also called Log-distance path loss model) is used and it is modelled by [?]. In this proposal, the losses given by the environment are estimated following equation 1.

$$
P L(d)[d B]=\overline{P L}\left(d_{0}\right)+10 n \log \left(\frac{d}{d_{0}}\right)+\sum L+\psi_{d B}
$$

where $P L(d)[d B]$ is the total signal attenuation as a function of the distance between the sensor and the sink node given in decibels, $\overline{P L}\left(d_{0}\right)$ is the signal attenuation at a distance $d_{0}$ from the sink, determined through measurements or through Equation 2 presented next, $n$ is the path loss exponent, $\sum L$ are the sumatory of looses given in the environment, and $\psi_{d B}$ represents the shadowing effect and it is given by a Gaussian random variable with zero mean and standard deviation $\sigma_{d B}$.

$$
\overline{P L}\left(d_{0}\right)=20 \log \left(\frac{\lambda}{4 \pi d_{0}}\right)
$$

In Equation 2, $\lambda$ is the transmission wavelength, which is $125 \mathrm{~mm}$ for the considered frequency of $2.4 G H z$, and through this method $\overline{P L}\left(d_{0}\right)=-40.0460[d B]$.

Given the considered signal loss causes, the received signal power can be calculated by

$$
P_{r}(d)[d B m]=P_{t}[d B m]-P L(d)[d B]
$$

where $P_{r}(d)[d B m]$ is the received power in $d B m$ as a function of the distance between transmitter and receiver, $P_{t}[d B m]$ is the transmitted power in $d B m$, and $P L(d)[d B]$ are the losses as a function of the distance, given by Equation 1.

Although the sensors are considered to be static, i.e., they are deployed somewhere and they do not move along the time when they leave the network or their batteries are exhausted, the shadowing part is still considered, but it is randomly selected when the node becomes active and then kept constant for the rest of the sensor lifetime. 


\section{A. Losses given by the environment}

The initial statement of this proposal is to apply a WSN for military purposes, thus it can be used in any type of environment (indoor, indoor to outdoor, outdoor between buildings, outdoor in a rural area, etc.). Thus, there is a wide range of issues that may affect to the loss of signal. Depending on the environment where the WSN is deployed, external losses may be very different. In this subsection, the parameter that takes into account the losses produced by objects placed in the environment (walls, floors, furniture, trees, etc.) and by the factors inherent of the environment (humidity, temperature, sandstorm, etc.) are discussed.

On one hand, if indoor environments are consider, the issues that will mainly affect to the loss of the signal are the walls, windows and floors of the buildings [?] [?]. They mainly depend on the building constructions materials. Taking into account that there could be different types of walls (e.g. a wall of a toilet may have higher loss than the one caused by the rest of the walls in the building because of the pipes embedded inside them), floors (depending on the building construction), and windows in the straight-line, these losses can be estimated following next equation.

$$
L[d B]=\sum\left(K_{i} F_{i}\right)+\sum\left(G_{j} H_{j}\right)+\sum\left(I_{k} W_{k}\right)
$$

where $K_{i}$ is number of floors of kind $i$ in the propagation path and $F_{i}$ is attenuation of one floor of kind $i, G_{j}$ is the number of walls of kind $j$ in the propagation path and $H_{j}$ attenuation factor of one wall of kind $j$, and $I_{k}$ is the number of windows of kind $k$ in the propagation path and $W_{k}$ attenuation factor of one windows of kind $k$. The equation includes having different types of wall, floors, and windows.

This equation only considers the walls, floors and windows, but the furniture or any type of object may be intersected in the straight-line. These looses are taken into account in many indoor wireless designs [?].

It was estimated in previous works that there was a mean wall loss of $6.29[\mathrm{~dB}]$ in 2 square kilometer campus with 50 buildings [?]. The mean wall loss value for those buildings changes from $4.66[\mathrm{~dB}]$ to $8.21[\mathrm{~dB}]$. it was also observed that loss can be as great as $20[\mathrm{~dB}]$ in toilet walls. Additionally, it must be taken into account the metallic objects (fences, statues, etc.) in the direct path. In these cases, obtained measures suffer errors above $2[\mathrm{~dB}]$. Depending on the 


\begin{tabular}{|l|c|c|}
\hline \multirow{5}{*}{ Indoor } & Environment & Path Loss typical values \\
\hline & Wall loss & $4.66[\mathrm{~dB}]-8.21[\mathrm{~dB}]$ \\
& Objects (fences, statues, etc.) & (toilet wall: approx. $20[\mathrm{~dB}]$ ) \\
& Floor loss & $12.9[\mathrm{~dB}]-35.4[\mathrm{~dB}]$ \\
\hline \multirow{5}{*}{ Outdoor } & Inside to outside building & $10[\mathrm{~dB}]-11.4[\mathrm{~dB}]$ \\
& Suburban environments & $5[\mathrm{~dB}]-16[\mathrm{~dB}]$ \\
& High rain intensity $(150 \mathrm{~mm} / \mathrm{hr})$ & $0.02[\mathrm{~dB} / \mathrm{Km}]$ \\
& Vense tree environment & $1.2[\mathrm{~dB} / \mathrm{m}]$ \\
& Sandstorm & $8.2[\mathrm{~dB}]-10.6[\mathrm{~dB}]$ \\
& & $10[\mathrm{~dB}]-26[\mathrm{~dB}]$ \\
\hline
\end{tabular}

TABLE I

LOSSES GIVEN BY THE ENVIRONMENT.

building construction material, the floor attenuation may change from $12.9[\mathrm{~dB}]$ to $35.4[\mathrm{~dB}][$ ?] Floors have the highest attenuation factor. Thus it should be taken into account when designing WSNs in this environment. These losses can be also taken into account when the communication is between two devices, one inside the building and the other outside the building. There are some studies that estimate these losses between $10[\mathrm{~dB}]$ and $11.4[\mathrm{~dB}]$ for $2.4 \mathrm{GHz}$ [?]. Moreover the shadow fading term obtained in suburban environments is estimated between 5-6[dB] [?].

Table I shows a comparison of losses given by the aforementioned environments.

\section{B. Shadowing Process Standard Deviation}

The standard deviation of the shadowing process $\sigma_{d B}$ has also been estimated in some experimental work. Similarly to the path loss exponent, this parameter is also dependent on the considered scenario. Nevertheless, less details are given in the literature regarding this parameter. Castiglione et al. [?] have characterized an indoor channel based on empirical data, achieving the value $\sigma_{d B}=4.4[d B]$ for fixed nodes, and $4.6[d B]$ for mobile nodes. In an extension of their work, more experiments have been conducted at the Stanford University campus, and standard deviations of $7.96[d B]$ and $7[d B]$ have been found [?]. Although Khan et al. [?] main focus was not on fading parameters, they have investigated the effects of the fading standard deviation on the connectivity of a WSN. In this work, they have considered standard deviations of $0[d B]$, 
$6[d B]$, and $8[d B]$. Once more, for indoor communication, Oestges et al. [?] have derived the value $4.43[d B]$ for the shadowing standard deviation, and they have considered $8[d B]-10[d B]$ for outdoor-to-indoor communication referring to previous work. Finally, gathering values from the literature, Goldsmith has found values from $5[d B]$ to $12[d B]$ for $\sigma_{d B}$ depending on the considered environment and system [?]. Thus, from the results achieved by previous research, it can be seen that indoor communication present a lower shadowing standard deviation than outdoor systems. In order to comprise the range of values referred in the literature, nevertheless keeping results presentation readable, in this work $\sigma_{d B}$ will be considered to change from 0 to $13[d B]$ in $1[d B]$ steps.

\section{Signal-to-Noise Ratio}

Since the considered multiple access method is TDMA, only one device is supposed to transmit at a time, and thus there is no transmission interference. Then, the bit error rates (BERs) will only be affected by channel losses (path loss and shadowing) and channel noise.

Considering the channel to present additive white Gaussian noise (AWGN), the noise power (or the noise variance) $\sigma_{A W G N}^{2}$ at the output of the receiver filter can be given by (adapted from $[?])$

$$
\sigma_{A W G N}^{2}=g \cdot \frac{N_{0}}{2} \cdot B
$$

where $g$ is the filter gain (considered to be 1 ), $N_{0}$ is the noise spectral density, and $B$ is the receiver filter bandwidth. The noise density is related to the receiver equivalent temperature through the equation

$$
N_{0}=k \cdot T
$$

where $k$ is the Boltzmann constant $\left(1.3806504 \cdot 10^{-23}[\mathrm{~J} / \mathrm{K}]\right)$ and the temperature $K$ is considered to be $293.15[\mathrm{~K}]$.

For IEEE 802.15.3 nodes, it can be seen from the standard that the receiver filter bandwidth is 15 [MHz] and for IEEE 802.15.4, it is 2 [MHz]. Thus, from the above equations and considerations, one can calculate the noise power $\sigma_{A W G N}^{2}=30.3553 \cdot 10^{-15}[\mathrm{~W}]=-105.1776512[\mathrm{dBm}]$ for IEEE 802.15.3 and $\sigma_{A W G N}^{2}=4.0474 \cdot 10^{-15}[\mathrm{~W}]=-113.9283[\mathrm{dBm}]$ for IEEE 802.15.4. 


\section{Bit Error Rates}

Quadrature phase-shift keying (QPSK) is the modulation scheme used by IEEE 802.15.3 compliant nodes. The bit error rate for transmission using QPSK is given by [?]

$$
B E R=\frac{1}{2} \cdot \operatorname{erfc}\left(\sqrt{\frac{E_{b}}{N_{0}}}\right)
$$

where the ratio $E_{b} / N_{0}$ is the ratio between the energy per bit and the noise spectral density. Given the noise power after the receiver filter $\left(\sigma_{A W G N}^{2}\right)$, the received signal power $\left(P_{r}\right)$, the filter bandwidth $(B)$, and the transmission rate $\left(R_{b}\right)$, this ratio is given by

$$
\frac{E_{b}}{N_{0}}=\frac{P_{r}}{\sigma_{A W G N}^{2}} \cdot \frac{B}{R_{b}} .
$$

IEEE 802.15.4 nodes transmit with offset QPSK (O-QPSK) and its bit error rate is also given by Equation 7 [?]. According to the aforementioned parameters for each technology, the BERs are shown in Figure 2 for SNRs from 0.01 [dB] to 12 [dB].

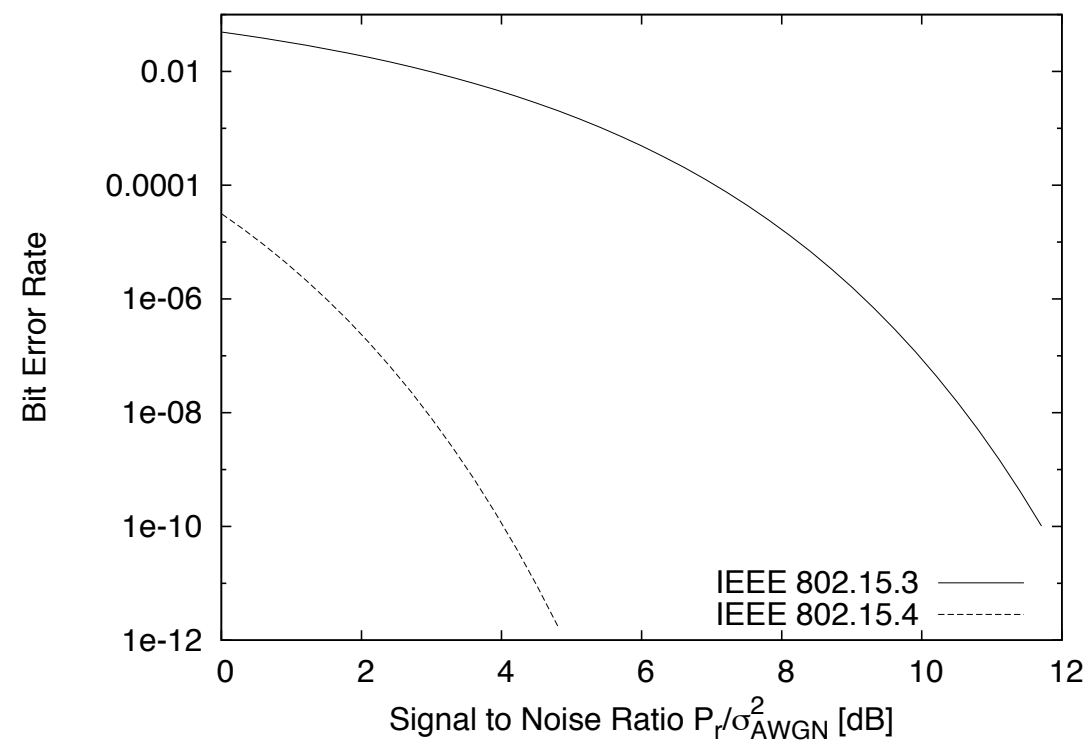

Fig. 2. Bit error rates for IEEE 802.15.3 and IEEE 802.15.4.

In this work, the minimum BER required to enable a node to associate to the network is considered to be around $10^{-8}\left(P_{r} / \sigma_{A W G N}^{2}>3 d B\right)$ for IEEE 802.15.4 and around $10^{-6}$ $\left(P_{r} / \sigma_{A W G N}^{2}>9 d B\right)$ for IEEE 802.15.3 nodes. 


\section{E. Physical Layer Considerations Regarding Simulation}

The simulation of variable channel characteristics and detailed physical layer behavior usually requires the use of additional simulation tools like Matlab or specific simulators built from scratch using an arbitrary programming language. In addition, communicating the results obtained from these tools with the used network simulator is a complex task [?]. In order to consider the channel characteristics in the carried out simulations, the shadowing loss is randomly chosen when a new node joins the network and it stays constantly up to the time when the node leaves the network. This procedure accounts for the effects of nodes placed in areas with many obstacles between the node and the sink as much as for the effects of nodes placed in line of sight with the sink node.

Moreover, as the channel is considered to be AWGN, the BERs regarding noise, free space attenuation, and shadowing will be calculated and compared to predefined thresholds for each type of traffic. Stricter delay requirements will be applied to Real Time traffic, however their BER threshold will be less strict than the one considered for BE traffic. On the other hand, there will not be any delay requirements for BE traffic, but the received signal BER has to be lower. Considering these differences, it is expected that nodes generating BE traffic will have a higher connection success rate than the ones generating Real Time traffic.

\section{Dynamic Time Division Multiple Access}

\section{A. Superframe Structure}

In order to provide support for both IEEE 802.15.3 and IEEE 802.15.4 nodes simultaneously, the TDMA superframe includes interleaved periods reserved for each type of node, thus requiring a sink compliant with both standards. The considered superframe is depicted in Figure 3.

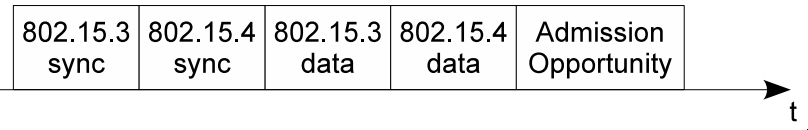

Fig. 3. TDMA superframe with support for IEEE 802.15.3 and IEEE 802.15.4.

The frames sent on each technology phase are considered to have maximum payload sizes in order to make synchronization easier. These frames are depicted in Figure 4 (IEEE 802.15.4) 
and Figure 5 (IEEE 802.15.3).

\begin{tabular}{|c|c|c|c|c|c|c|c|c|}
\hline \multirow[t]{2}{*}{ Octets: } & 2 & 1 & 0 & 0 & 2 & 2 & 102 & 2 \\
\hline & $\begin{array}{l}\text { Frame } \\
\text { Control }\end{array}$ & $\begin{array}{c}\text { Sequence } \\
\text { Number }\end{array}$ & $\begin{array}{l}\text { Destination } \\
\text { PAN ID }\end{array}$ & $\begin{array}{c}\text { Destination } \\
\text { Address }\end{array}$ & $\begin{array}{l}\text { Source } \\
\text { PAN ID }\end{array}$ & $\begin{array}{c}\text { Source } \\
\text { Address }\end{array}$ & Data Payload & FCS \\
\hline
\end{tabular}

Fig. 4. IEEE 802.15.4 MAC frame.

\begin{tabular}{|c|c|c|c|c|c|c|c|c|}
\hline \multirow[t]{2}{*}{ Octets: } & 2 & 2 & 1 & 1 & 3 & 1 & 2044 & 4 \\
\hline & $\begin{array}{l}\text { Frame } \\
\text { Control }\end{array}$ & $\begin{array}{l}\text { Piconet } \\
\text { ID }\end{array}$ & $\begin{array}{l}\text { Destination } \\
\text { ID }\end{array}$ & $\begin{array}{c}\text { Source } \\
\text { ID }\end{array}$ & $\begin{array}{c}\text { Fragmentation } \\
\text { Control }\end{array}$ & $\begin{array}{l}\text { Stream } \\
\text { Index }\end{array}$ & Data Payload & $\mathrm{FCS}$ \\
\hline
\end{tabular}

Fig. 5. IEEE 802.15.3 MAC frame.

All phases except admission opportunity will have dynamic sizes. Nodes successfully admited in the network will send one frame with maximum payload during their corresponding technology data phase. The admission opportunity is divided in fixed time subphases to detect and wait for the response of nodes compliant with each technology, as shown in Figure 6.
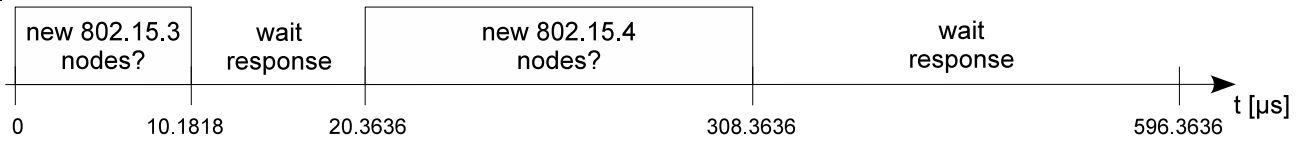

Fig. 6. Admission opportunity time subframe (time relation out of scale).

\section{B. Synchronism}

The synchronization time subframes will be broadcasted and received only by the compliant nodes, since different channels will be used for IEEE 802.15.3 nodes and IEEE 802.15.4 nodes. Upon reception of such subframes, each node will have information about the current time, the next superframe start time, the number of IEEE 802.15.3 compliant nodes associated to the network and their transmission order, and the number of IEEE 802.15.4 associated nodes and their transmission order. This information is distributed in the data payload field of IEEE 802.15.3 or IEEE 802.15.4 according to the current synchronization subphase, as shown in Figure 7. 


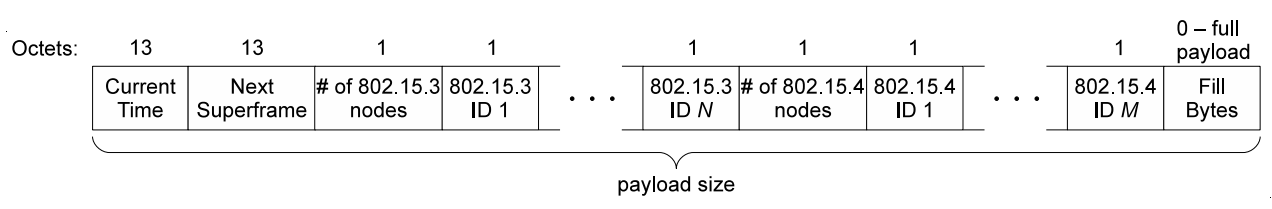

Fig. 7. Syncrhonism frame for both IEEE 802.15.3 and IEEE 802.15.4 considering a network with $N$ IEEE 802.15.3 associated nodes and $M$ IEEE 802.15.4 associated nodes.

In order to avoid synchronization disruption because of the variable size of synchronization messages, these frames will always be transmitted with the full length of the payload field (filled as necessary) according to each technology maximum frame size.

\section{Cross-Layer Design}

The nodes with IEEE 802.15.3 characteristics, responsible for transmitting multimedia in the defined scenario, are considered to have frame transmission delay limits that should be respected by the TDMA dynamic frame or the multimedia data may not be relevant for the receiver. Thus, the sink node cannot allow every new arriving node to associate to the network and receive a TDMA slot in order to respect the multimedia streams delay limits. Hence, a cross-layer node admission control is proposed in order to keep the TDMA superframe inside the allowed boundary by using the application layer maximum frame delay information at the link layer. Moreover, it allows us to incorporate several types of traffic classes by adding different privileges to the frames. The new interface to transmit this information is depicted by the dashed arrow in Figure 8.

The cross-layer proposal requires to make some changes to the IEEE 802.15.3 and 802.15.4 nodes. Since both node technologies can operate with CSMA/CA and TDMA phases [?] [?], a minor modification should be done to remove CSMA/CA and extend TDMA to cope with the proposed superframe. However, a major requirement for the operation of the cross-layer proposal is the usage of a sink node capable of communicating with both types of nodes. This central node should be created since, to the best of the authors knowledge, there is no commercial device that can cope with both standards. It is not only the major requirement of the proposal, but also its drawback. The costs, both in terms of money as of time, are high for a research team, and this work alone could result in a new research paper. Nonetheless, the advantages of 


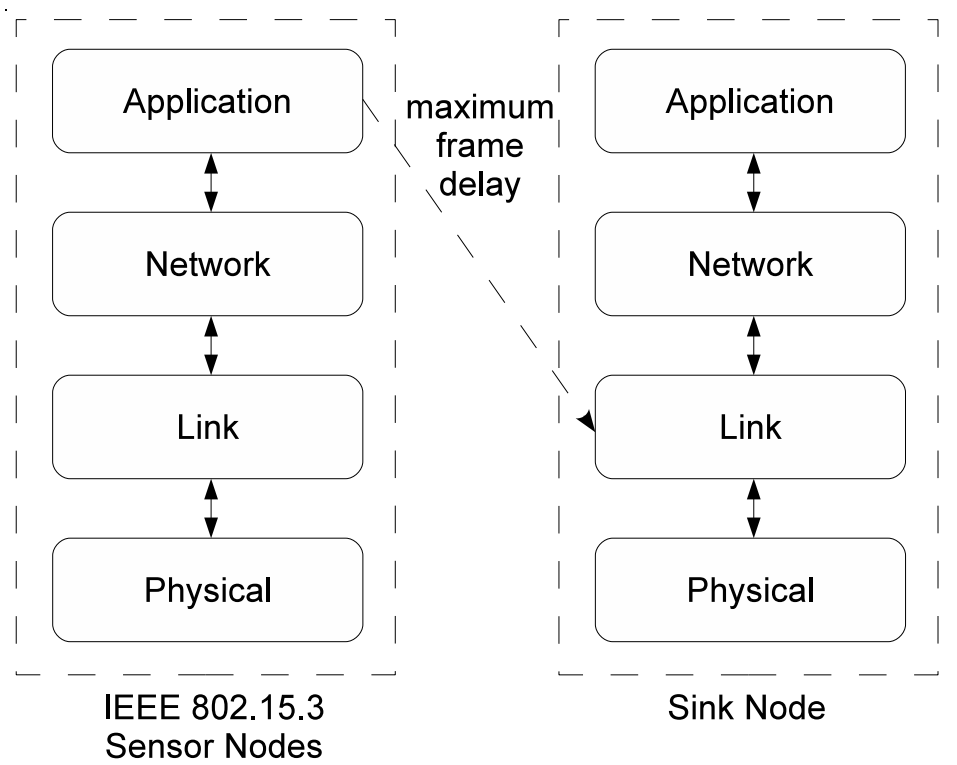

Fig. 8. Considered cross-layer design.

the proposal are that sensors energy will be saved by entering sleep node when it is not their turn to transmit and that the necessary QoS will be always mantained for the multimedia streams from IEEE 802.15.3 nodes.

\section{Error Control}

The use of automatic repeat request (ARQ) is not needed for either of the considered traffic classes. From the traffic classes point of view, best effort traffic is supposed to cope with segment losses and for real-time traffic it is better to lose a segment than to retransmit it. For the latter kind of traffic, segments need to be timely delivered, and retransmission will disrupt this capability. Nevertheless, forwarding error correction (FEC) could add bit error protection to the transmitted frames, even though its use adds transmission overhead. In order to reduce the complexity of the presented analysis, FEC will not be considered here as well. This method might be considered in future work.

\section{Performance Evaluation}

The dynamic TDMA protocol with cross-layer admission control has been deployed using the OMNeT++ simulator [?]. New nodes are created in intervals according to an exponential random 
generator for each type of node considering the same mean. When a node is created, the path loss calculations considering random shadowing are carried out, and if at least the minimum signal to noise ratio is achieved, the new node is considered eligible to connect to the sink. Then, these arriving nodes are put in a list corresponding to the node technology. These lists are verified during the admission opportunity phase, and in case there is more than one node in the list, which means that there will be more than one answer to the "new 802.15.X nodes?" question, a collision occurs and the collided nodes need to be redeployed. If no collision occurs during the admission opportunity phase, then the maximum frame delay of the multimedia nodes is verified in order to decide whether the new node can be admitted or not. Finally, if the delay limits are still respected for the admission of the new node, it receives its schedule at the "sync" phase, and can transmit frames until its batteries are exhausted.

In order to evaluate the influence of the nodes interarrival time, the assumptions made for the simulations are presented next. The considered network size was $500 \mathrm{~m}$ x $500 \mathrm{~m}$, as shown in Figure 1, the shadowing standard deviation $\left(\sigma_{d B}\right)$ was $9 d B$, the path loss exponent $(n)$ was 2.8 , the transmission power was $0 \mathrm{dBm}(1 \mathrm{~mW})$, the multimedia frame delay limit was set to $60 \mathrm{~ms}$, and the nodes mean interarrival varied logarithmically from $1 \mathrm{~ms}$ to $1 \mathrm{~s}$. The above- mentioned simulation parameters are summarized in Table II. The results achieved for both technologies blocking $\left(P_{B}\right)$, collision $\left(P_{C}\right)$, connection failure $\left(P_{F}\right)$, and connection success $\left(P_{S}\right)$ probabilities are shown in Figure 9.

TABLE II

SIMULATION PARAMETERS.

\begin{tabular}{cc}
\hline Simulation parameter $($ symbol) & Value \\
\hline Network size & $500 \mathrm{~m}$ \\
Shadowing standard deviation $\left(\sigma_{d B}\right)$ & $9 d B$ \\
Transmission power $\left(P_{t x}\right)$ & $0 d B m(1 \mathrm{~mW})$ \\
Path loss exponent $(n)$ & 2.8 \\
Multimedia frame delay limit & $60 \mathrm{~ms}$ \\
Node mean interarrival time & $1 \mathrm{~ms}-1 \mathrm{~s}$ \\
\hline
\end{tabular}

The first fact that can be noticed from Figure 9 is the constant connection failure probabilities for both technologies $\left(P_{F}(3)\right.$ and $\left.P_{F}(4)\right)$. Since these probabilities depend on the network size, the shadowing process, and the path loss exponent, they have not changed with the node 


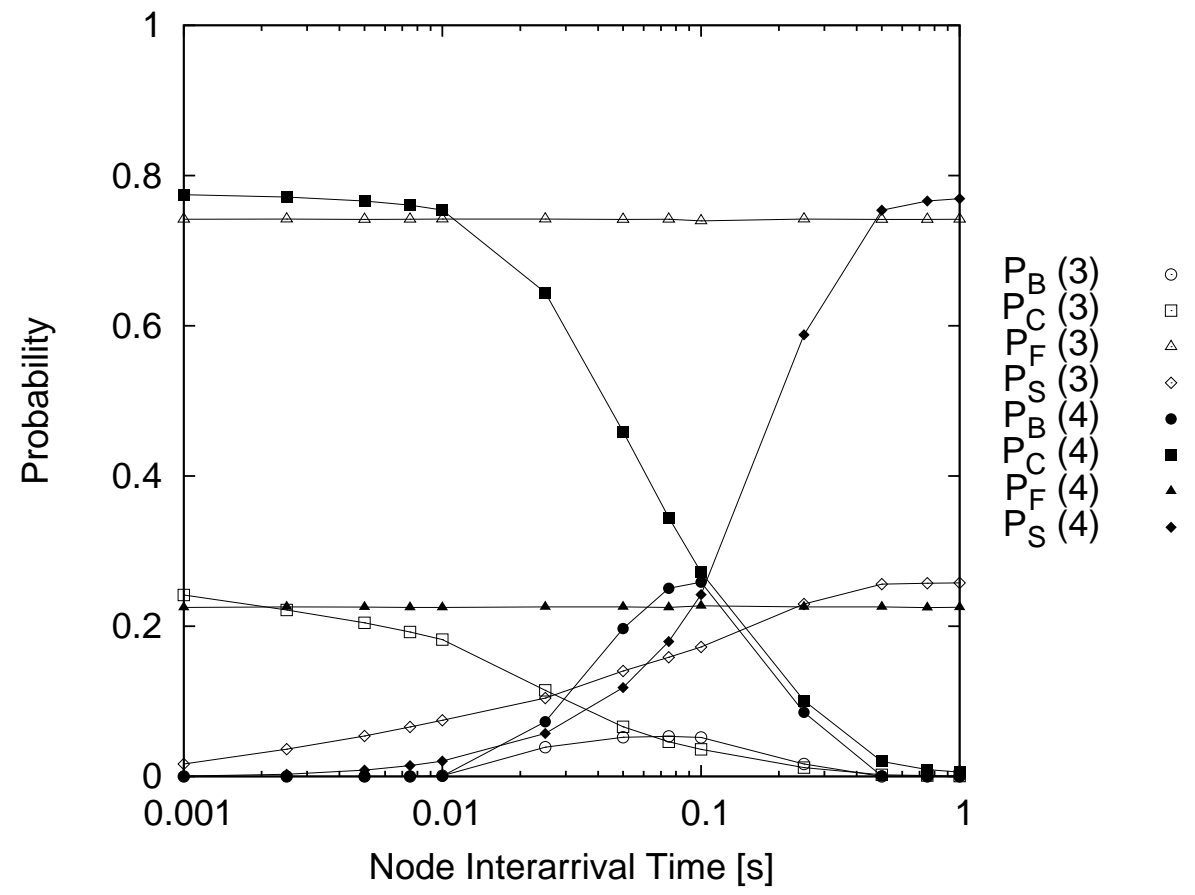

Fig. 9. Blocking probabilities $\left(P_{B}\right)$, collision probabilities $\left(P_{C}\right)$, connection failure probabilities $\left(P_{F}\right)$, and connection success probabilities $\left(P_{S}\right)$ for IEEE 802.15.3 (3) and IEEE 802.15.4 (4) compliant nodes.

interarrival time. Moreover, the failure probability for multimedia nodes is more than three times the probability for scalar sensors. This happens because all sensors transmit with the same power and, as shown previously, IEEE 802.15.3 requires more power to achieve the same bit error rates of IEEE 802.15.4 nodes. It can also be seen that, since most of the multimedia nodes failed to establish communication with the sink, its collision probability is much lower than the collision probability for the scalar sensors. Moreover, as the time between the arrival of new nodes increases, both collision probabilities $\left(P_{C}(3)\right.$ and $\left.P_{C}(4)\right)$ decrease because the probability of arrival of two or more nodes during the same superframe also decreases. Moreover, the behavior of the blocking probabilities is different from the others. The nodes that have not failed to connect and have not collided are subject to be blocked. For shorter interarrival times than $0.1 s$, the blocking probabilities increase with the interarrival time because less nodes collide, and thus more nodes attempt to obtain a transmission time slot. For longer interarrival times than $0.1 s$, the blocking probabilities decrease because admitted nodes have enough time to exhaust their batteries and leave the network, leaving time slots for incoming nodes. Finally, the success 
probabilities are increasing with the node interarrival time because of their dependency on the other probabilities. It is given by

$$
P_{S}=1-\left(P_{B}+P_{C}+P_{F}\right)
$$

The same parameters have been used to gather data on the mean superframe duration and its standard deviation as a function of the nodes interarrival time. The results are shown in Figure 10.

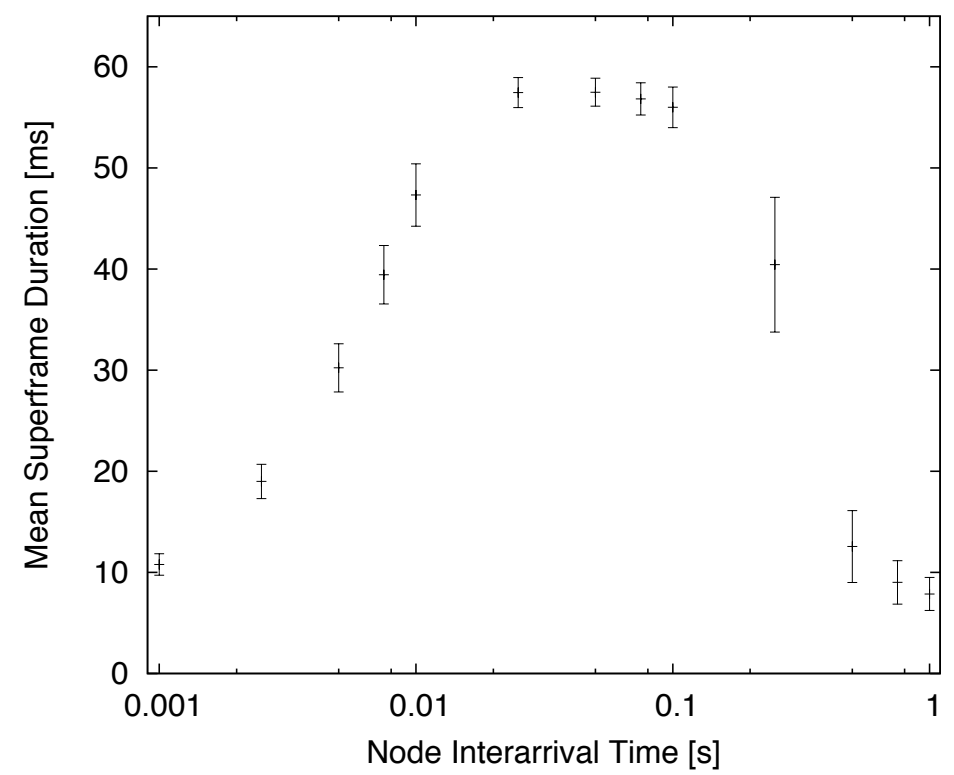

Fig. 10. Superframe mean duration and standard deviation (error bars) as a function of the nodes interarrival time.

In Figure 10, it can be seen that the mean superframe duration has a curve behavior that resembles the blocking probability curves behavior shown previously in Figure 9. Thus, it can be inferred that the superframe duration is prevented from reaching its maximum value of $60 \mathrm{~ms}$ - the multimedia nodes time constraint - by the same reasons pointed out before. For shorter nodes interarrival time than $0.01 \mathrm{~s}$, collisions prevent new nodes from associating, while for larger interarrival time than $0.1 s$, there are less nodes trying to associate than the TDMA schedule is able to serve. It should be interesting to keep the superframe duration as close to its limit as possible in order to make better use of the available TDMA slots. 
Since the connection failure probabilities do not depend on the nodes' interarrival time, their variation has been assessed as a function of the path loss exponent and as a function of the shadowing random process standard deviation. First, the path loss exponent $(n)$ is varied in the range from 1.8 to 3.8 in steps of 0.2 , and then, the shadowing process standard deviation is varied from $0 d B$ to $13 d B$ in steps of $1 d B$. The simulation results for each case are shown in Figures 11 and 12, considering mean nodes' interarrival time of $0.05 \mathrm{~s}$.

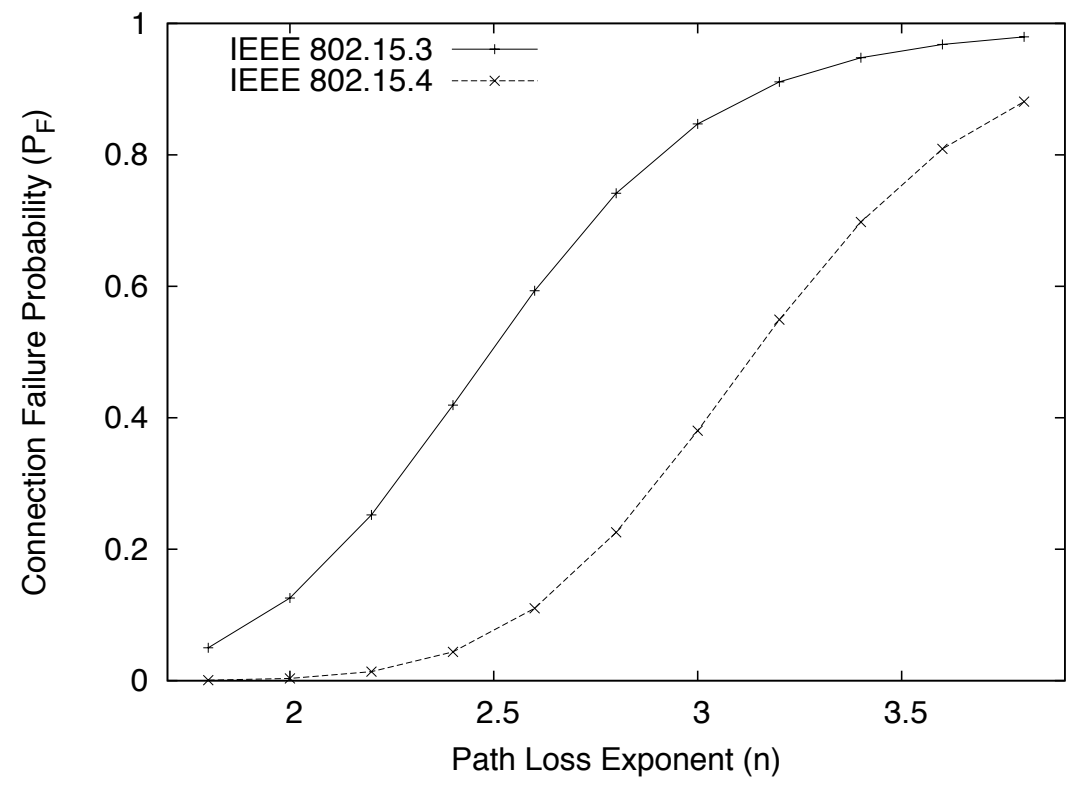

Fig. 11. Connection failure probabilities as a function of the path loss exponent.

As expected, from Figure 11, it can be seen that the failure probabilities increase for both technologies with the increase of the path loss exponent. Thus, the more cluttered the environment, the higher the chances to fail to connect to the sink node. Also, as the multimedia nodes are more sensitive to the channel effects, their connection failure probabilities are greater than the probabilities for the scalar sensors.

In Figure 12, the connection failure probability variation is shown as a function of the shadowing process standard deviation.

It can be seen from Figure 12 that the connection failure probability increases as the shadowing standard deviation increases for IEEE 802.15.4 nodes, but on the other hand, it decreases this probability for IEEE 802.15.3 nodes. 


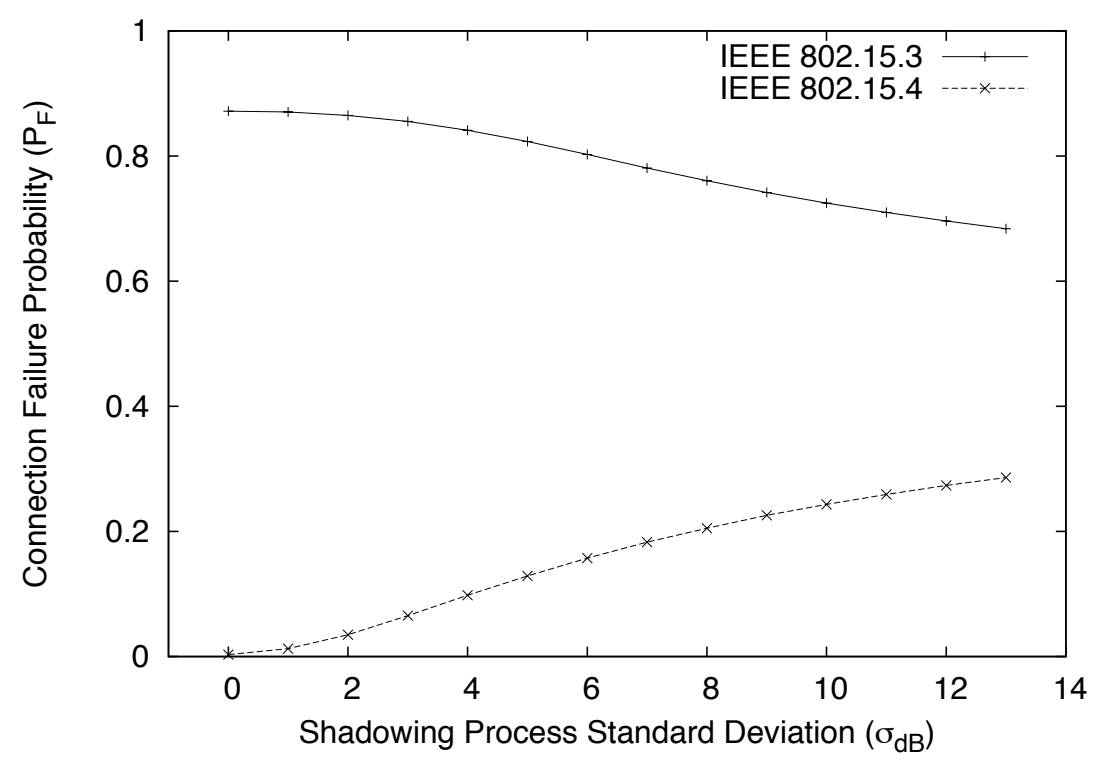

Fig. 12. Connection failure probabilities as a function of the shadowing process standard deviation.

It can be explained by the dominance of the shadowing process on the randomness of the connection failure probability over the random deployment process. Because nodes are deployed at random places inside the network limits, thus also making random the distance between the sensor nodes and the sink (at the center of the network), one might expect that both deployment and shadowing will have effects on the connection failure probability. This effect is shown in Figure 13 by the shaded areas.

It can also be seen in Figure 13 that for some small variation of the distance for each technology, the connection failure probability is greater for IEEE 802.15.3 than for IEEE 802.15.4 nodes. Furthermore, it is clear that the node distance has impact on the probability values, which is depicted as a greater shaded area as the curves move left. It is worth noting that the bellshaped curves represent the shadowing process, which is modulated by the random deployment process. Thus, exaggerating the shadowing process standard deviation and using the same node distances, the probabilities of yielding a determined signal to noise ratio at the receiver changes to the ones depicted in Figure 14.

By comparing Figures 13 and 14, it is clear that the shaded areas, and thus the connection failure probabilites, are not as affected by the distance variation as they were in the first case. 


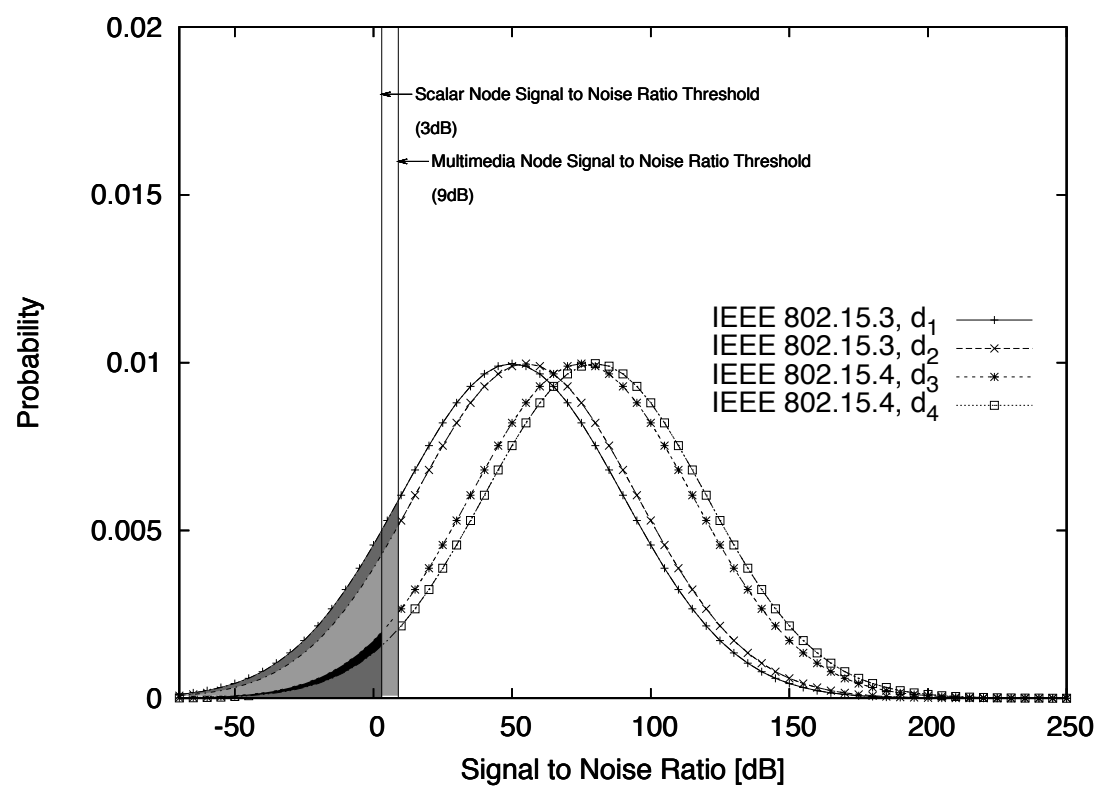

Fig. 13. Probability density function of the signal to noise ratio at the receiver as a function of the node technology deployed at two arbitrary distances from the sink; the shaded areas represent the connection failure probabilities for the given technologies and distances.

Moreover, it can be seen that both probabilities approach to the 0.5 value as the shadowing process standard deviation increases, which proves why the failure probabilities shown in Figure 12 tend to 0.5. Multimedia nodes can benefit from shadowing, while scalar nodes are impaired by this kind of fading.

\section{AdMISSION CONTROL SYSTEM COMPARISON}

As far as authors know, there is not any admission control system for IEEE 802.15.3 and IEEE 802.14.4 based on a cross-layer approach such as the one presented in this paper. This crosslayer proposal is based on a central node that is able to communicate with different nodes. The MAC protocol can adapt itself to enable the communication of incoming and outgoing nodes, respecting some predetermined QoS metrics. Now, the admission control mechanisms for IEEE 802.15.3 and IEEE 802.14.4 available in the literature are introduces and they will be compared with the proposal presented in this paper.

Admission control mechanisms have special interest in many types of wireless networks. Yang Xiao et al. presented in [?] an admission control approach for QoS support in wireless ad hoc 


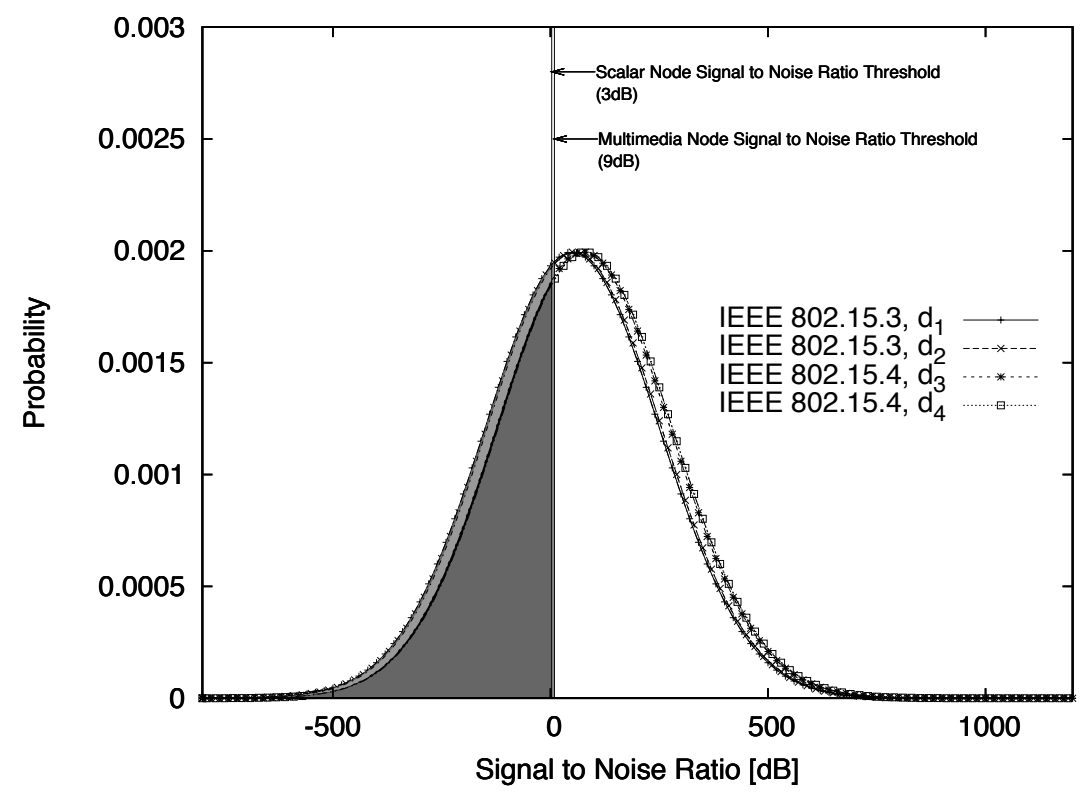

Fig. 14. Probability density function of the signal to noise ratio at the receiver as a function of the node technology deployed at two arbitrary distances from the sink, with exaggerated shadowing standard deviance; the shaded areas represent the connection failure probabilities for the given technologies and distances.

networks. They proposed two local data-control schemes and an admission-control scheme for ad hoc networks using IEEE 802.11e MAC standard. The proposed distributed admission-control scheme is based on their previous measurements. Each node decides the acceptances/rejections of flows without the need of access points. Their study shows that the combination of the local data control schemes with the admission control scheme allows to guaranteed QoS under a clear channel condition while maintaining a good utilization. Moreover, Yuechun Chu and Aura Ganz evaluated and compared several MAC protocols for UWB-based wireless networks [?] in terms of throughput, admission ratio and energy consumption. They highlighted the need of admission control and observed that for both, QoS traffic and Best Effort traffic, the centralized protocol achieves a better admission ratio and higher throughput than distributed protocols. In distributed protocols, the cooperative distributed protocol obtains a better admission ratio than the non-cooperative distributed protocol.

One of the oldest papers about admission control for IEEE 802.15 is the one presented by Jelena Misic in 2004 [?]. In this paper, the authors proposed three admission algorithms for Bluetooth piconets. One of them was based on queue stability, which was the best one for 
battery power-limited masters, another one was based on estimating the access delay of the slave upon admission, and the last one was based on the predefined cycle time bounds, which was the best one for applications that generate constant-bit-rate data flows.

In [?], Wei Sheng proposed a cross-layer admission control policy for a code-division-multipleaccess (CDMA) system in order to provide heterogeneous services with high data rate and guaranteed quality-of-service (QoS). Author developed an exact outage probability, which is then employed to derive the optimal call admission control policy, by formulating a constrained semi-Markov decision process, in order to maximize the system throughput with guaranteed QoS requirements in both physical and network layers. The system was neither focused on WSNs, but on general CDMA systems, nor in TDMA such as the one presented in this paper, but it shows the importance of such systems for wireless access to voice, data and multimedia traffic.

In [?], the authors proposed the use of cross-layer approaches to overcome WSNs constraints because they are the most efficient optimization techniques. A great number of cross-layer approaches have appeared for WSNs in order to address their problems. One of the main challenges is the QoS provisioning for multimedia purposes because of the WSN lifetime (due to the sensors energy consumption), thus it makes sense the use of cross-layer approaches for admission control in IEEE 802.15.3 and IEEE 802.15.4 networks.

Hang $\mathrm{Su}$ et al. applied cross-layer based battery-aware time TDMA MAC protocols for wireless body-area monitoring networks in Healthcare Applications [?]. Their proposed schemes can significantly increase the battery lifespan of sensor nodes while satisfying the reliability and delay-bound QoS requirements for wireless body-area monitoring networks, outperforming the IEEE 802.15.4 and Bluetooth protocols.

Khoa T. Phan et al. stated that node admission is essential for wireless multimedia sensor networks (WMSNs) in order to support multimedia services such as delivery of video and audio streams [?]. They proposed a cross-layer design based on two-stages. In the first stage the number of admitted sensor nodes is maximized, and in the second stage the network lifetime is maximized. Their proposal does not identify for which protocol it has been proposed.

Now, the admission control mechanisms for TDMA in IEEE 802.15.3 and IEEE 802.15.4 found in the related literature are presented.

WMSNs are being proposed for many application environments. Muhammad O. Farooq et al. proposed a cross-layer architecture for supporting multiple applications in order to support 
multiple applications on a single sensor node through the use of a separate memory area for each application [?]. Their proposal classifies WMSNs traffic into six classes. The framework uses a shared database to enable cross-layer interactions. They proposed a TDMA-based distributed MAC to support heterogeneous traffic flows in IEEE 802.15.4.

In [?], authors propose a synchronization mechanism based on Time Division Beacon/superframe Scheduling (TDBS), which efficiently assigns distinct time windows to adjacent clusters in cluster-tree WSNs, in order to avoid inter cluster frame collisions (beacons and data). Moreover, they present an approach for efficiently managing duty-cycles in every cluster. This approach ensures the fairest use of bandwidth resources. The feasibility of their proposal is demonstrated via an experimental test-bed involving a cluster-tree WSN.

A. Rangnekar et al. proposed a QoS-aware, multi-channel scheduling algorithm that simultaneously utilizes the various channels available in UWB based networks for IEEE 802.15.3 WPANs [?]. Their aim was to satisfy the increasing demand for higher bandwidth in order to support high data rate multimedia applications. The scheduling algorithm employs a distributed dynamic channel allocation algorithm to efficiently allot channels to neighboring, interfering piconets. It also differentiates between the various connections by allocating slots to each connection based on its access category.

In order to improve the scheduling model specified in IEEE 802.15.4, [?] Mishra et al. proposed a Guaranteed Time Slots (GTS) scheduling algorithm for LR-WPAN start topology in beacon-enabled mode that tries to meet the delay constraints of time-sensitive transactions. The drawback of the algorithm is that it may waste a portion of the GTS since it always allocates the GTSs from the first slot of the Contention-Free Period (CFP).

LA-MAC, an UWB-based location-aided MAC protocol for WSNs, was proposed by Eirini Karapistoli et al. in [?]. It is a random access MAC protocol with dedicated procedures for interference estimation. LA-MAC protocol exploits the accurate position information provided by the UWB transmission technique in order to assess the channel conditions more accurately and enhance the network performance. It improves the channel spatial reuse efficiency and throughput, allowing simultaneous data transmissions. The produced interference estimation model in conjunction with the distributed admission control scheme, leads to better interference predictions and blocking assessments.

In [?], JunKeun Song et al. propose a QoS guaranteed algorithm for real-time applications in 
IEEE 802.15.4 protocol. The proposed algorithm allocates a new guaranteed time slot dynamically to support real-time applications which have periodic messages in the LR-WPAN. It also decreases the drop rate occurred by the overflow of the queue when many messages arrive at the same time. Their simulations show that their slot allocation algorithm enables guaranteed services to be more efficient, and improves the bandwidth utilization, compared with IEEE 802.15.4 standard.

Table III compares this proposal with available admission control systems for IEEE 802.15.3 and IEEE 802.15.4 protocols. They were compared in terms of the following issues:

- The IEEE 802.15 variant where the proposal has been applied.

- The topology used in the proposal.

- If the proposal approach uses superframe extension.

- If the proposal is focused on Quarantee QoS or Multimedia.

- In which technique the proposal is based on.

- If the proposal is analytical or if it has been also simulated.

- If the proposal is designed bearing in mind traffic classes.

- If the proposal uses cross-layer approaches.

This comparison shows this proposal is the one with most features and the only one that can be applied to both IEEE 802.15.3 and IEEE 802.15.4.

\section{ViI. CONClusion And Future Work}

In this work, channel characterization scheme combined to a cross-layer admission control in a dynamic cloud-based multimedia sensor networks to share the network resources between any two nodes was proposed. The analysis shows the behavior of two nodes using different network access technologies and the channel effects for each technology. Moreover, it is also shown the existence of optimal node arrival rates in order to improve the usage of dynamic admission control when network resources are used. Extensive simulations are provided to validate the efficiency of the proposed dynamic admission control for cloud-based multimedia sensor networks. Future work might comprise the evaluation of CSMA and other medium access methods proposed in the literature. Moreover, different scenarios could be included, as well as different variations of nodes interarrival times for each technology. 


\begin{tabular}{|c|c|c|c|c|c|c|c|c|}
\hline Proposal & $\begin{array}{c}\text { IEEE } 802.15 \\
\text { Variant }\end{array}$ & Topology & $\begin{array}{l}\text { Superframe } \\
\text { Extension }\end{array}$ & $\begin{array}{c}\text { Focused on QoS } \\
\text { or Multimedia }\end{array}$ & Based on & $\begin{array}{l}\text { Type of } \\
\text { proposal }\end{array}$ & $\begin{array}{l}\text { Support for } \\
\text { Traffic classes }\end{array}$ & $\begin{array}{c}\text { Cross-layer } \\
\text { based }\end{array}$ \\
\hline $\begin{array}{l}\text { M. O. Farooq } \\
\text { et al. [?] }\end{array}$ & 802.15 .4 & Hexagon & Yes & Yes & bandwidth & Analytical & Yes & Yes \\
\hline TDBS [?] & 802.15 .4 & Cluster & Yes & No & Cycle time & $\begin{array}{l}\text { Analytical } \\
\& \text { simulation }\end{array}$ & No & No \\
\hline $\begin{array}{l}\text { A. Rangnekar } \\
\text { et al. [?] }\end{array}$ & 802.15 .3 & Random & Yes & Yes & $\begin{array}{c}\text { Cycle time } \\
\& \text { access category }\end{array}$ & $\begin{array}{l}\text { Analytical } \\
\text { \& simulation }\end{array}$ & No & No \\
\hline $\begin{array}{l}\text { J. Misic et al. } \\
\text { [?] }\end{array}$ & 802.15 .4 & Cluster & Yes & No & Packet service time & $\begin{array}{l}\text { Analytical } \\
\& \text { simulation }\end{array}$ & No & No \\
\hline ACS [?] & 802.15 .3 & Random & Yes & Yes & $\begin{array}{l}\text { Random access \& } \\
\text { superframe extens. }\end{array}$ & $\begin{array}{l}\text { Analytical } \\
\& \text { simulation }\end{array}$ & No & No \\
\hline $\begin{array}{l}\text { A. Mishra } \\
\text { et al. }[?]\end{array}$ & 802.15 .4 & Star & Yes & Yes & Transmission time & $\begin{array}{l}\text { Analytical } \\
\& \text { simulation }\end{array}$ & No & No \\
\hline LA-MAC [?] & 802.15 .4 & Random & No & No & $\begin{array}{l}\text { Interference } \\
\text { Indicator }\end{array}$ & $\begin{array}{c}\text { Analytical } \\
\& \text { simulation }\end{array}$ & No & No \\
\hline D-GTS [?] & 802.15 .4 & Random & Yes & Yes & Cycle time & Simulation & No & No \\
\hline This proposal & $\begin{array}{c}802.15 .3 \\
\& 802.15 .4\end{array}$ & Random & Yes & Yes & $\begin{array}{l}\text { Synchronization \& } \\
\text { Transmission delay }\end{array}$ & $\begin{array}{l}\text { Analytical } \\
\& \text { simulation }\end{array}$ & Yes & yes \\
\hline
\end{tabular}

TABLE III

COMPARISON TABLE.

\section{ACKNOWLEDGMENTS}

This work has been partially supported by Instituto de Telecomunicações, Next Generation

Networks and Applications Group (NetGNA), Portugal, and by National Funding from the FCT

- Fundação para a Ciência e Tecnologia through the Pest-OE/EEI/LA0008/2011. 